\title{
Analysis of miRNA expression profiles in breast cancer using biclustering
}

\author{
Antonino Fiannaca ${ }^{1 *}$, Massimo La Rosa², Laura La Paglia1, Riccardo Rizzo ${ }^{1}$, Alfonso Urso ${ }^{1}$ \\ From The 9th IAPR conference on Pattern Recognition in Bioinformatics \\ Stockholm, Sweden. 21-23 August 2014
}

\begin{abstract}
Background: MicroRNAs (miRNAs) are important key regulators in multiple cellular functions, due to their a crucial role in different physiological processes. MiRNAs are differentially expressed in specific tissues, during specific cell status, or in different diseases as tumours. RNA sequencing (RNA-seq) is a Next Generation Sequencing (NGS) method for the analysis of differential gene expression. Using machine learning algorithms, it is possible to improve the functional significance interpretation of miRNA in the analysis and interpretation of data from RNAseq. Furthermore, we tried to identify some patterns of deregulated miRNA in human breast cancer (BC), in order to give a contribution in the understanding of this type of cancer at the molecular level.
\end{abstract}

Results: We adopted a biclustering approach, using the Iterative Signature Algorithm (ISA) algorithm, in order to evaluate miRNA deregulation in the context of miRNA abundance and tissue heterogeneity. These are important elements to identify miRNAs that would be useful as prognostic and diagnostic markers. Considering a real word breast cancer dataset, the evaluation of miRNA differential expressions in tumours versus healthy tissues evidenced 12 different miRNA clusters, associated to specific groups of patients. The identified miRNAs were deregulated in breast tumours compared to healthy controls. Our approach has shown the association between specific sub-class of tumour samples having the same immuno-histo-chemical and/or histological features. Biclusters have been validated by means of two online repositories, MetaMirClust database and UCSC Genome Browser, and using another biclustering algorithm.

Conclusions: The obtained results with biclustering algorithm aimed first of all to give a contribute in the differential expression analysis in a cohort of BC patients and secondly to support the potential role that these non-coding RNA molecules could play in the clinical practice, in terms of prognosis, evolution of tumour and treatment response.

\section{Background}

MicroRNAs (miRNAs), among small non coding RNAs (sncRNAs), are emerging as key regulators in multiple cellular functions, due to their crucial role in many important physiological processes (cell development, cell proliferation, apoptotic process, and response to different external and stress signals) [1]. Moreover it has been recently demonstrated that miRNAs are differentially expressed in specific tissues, during specific cell statuses,

\footnotetext{
* Correspondence: fiannaca@pa.icar.cnr.it

${ }^{1}$ ICAR-CNR, National Research Council of Italy, viale delle Scienze, Ed.11, 90145 Palermo, Italy

Full list of author information is available at the end of the article
}

or in many diseases as tumours [2]. MiRNAs are small non coding molecules of single stranded RNA, 22-25 nt long, involved in the negative regulation of the gene expression to a post-transcriptional level. MiR-NAs in fact pair with specific RNA messengers (mRNA), causing their degradation or blocking the translational process of the protein product [3-5]. An interesting application involving these elements is the analysis of differential gene expression, obtained by comparing the transcriptional profiles of two or more individuals, tissues or cell types. Massive sequencing technique is an increasingly popular method used to study this mechanism. For instance, information on transcripts in healthy and 
diseased subjects may allow to identify which genes are expressed in a significantly different manner between these two groups and, thus, it is used to highlight differences that involve the pathological condition. Based on the evidence that groups of miRNAs are differentially expressed in several types of cancer, it is possible to study their involvement, negatively or positively, in the control of intracellular signalling pathways that have a direct influence on cancerous growth, such as proliferation and apoptosis. Therefore those miRNAs could act as oncogenes or tumour suppressors [3,6-11]. The importance of miRNAs in the regulation of gene expression is underlined also by the fact that few miRNAs can interact on many mRNAs. The altered expression of a single miRNA can lead to the deregulation of several molecular pathways, that may contribute separately, or cooperate, to the emergence of a malignant cell phenotype $[1,12,13]$. Many scientific works about differential gene expression analysis, show an association of miRNAs with development and tumour progression, as chronic lymphocytic leukemia (CLL), prostate cancer, non-small cell lung cancer (NSCLC), colorectal cancer (CRC) [2,7,14-17]. Volinia et al [18], reported a microarray analysis of miRNAs on 540 samples belonging to different tumour types (lung, breast, stomach, prostate, colon, pancreas), and showed a miRNA signature that join different solid tumours. Inside this signature were found miRNAs already known to be associated with other cancers, among them miR-17-5p, miR-20a, miR-21, miR-92, miR106a, and miR-155. In this last decade more than 400 studies focusing on the role of miRNA expression levels and their regulation in breast cancer have been published [19]. Unfortunately, most of these studies were focused only on a specific subset of miRNAs, or a limited group of patients $[20,21]$. The role of microRNAs was also demonstrated in the early stages of the disease progression and metastasis. In fact, several experimental evidences showed the involvement of miRNAs in the regulation of biological processes that lead to the acquisition of metastatic potential, including adhesion, invasion, migration, epithelial-mesenchymal transition (EMT) and angiogenesis [22,23].

In the past decade, the microarray technique was successfully used for the analysis of gene expression but, due to some limitations [24-26], more recently microarray technology was accompanied by RNA sequencing (RNA-seq) method for the analysis of the transcripts. RNA-seq method belongs to Next Generation Sequencing (NGS) techniques and generates DNA sequences complementary to the RNA reference molecule. With respect to microarray, two main advantages of RNA-seq method are (1) the ability to quantify a large dynamic range of expression levels, with absolute rather than relative values, and (2) the absence of a priori knowledge of the sequence to be analysed [27].

Nevertheless, there is not a standard method used to analyse the gene expression by RNA-seq data. In order to assist biologists in the complex task of interpretation of the large amount of data produced by NGS techniques, an investigation technique should be based on computational methods.

Clustering methods have been usually used to analyse gene expression data, with the purpose to discover groups of genes (clusters) that are co-regulated with respect to certain experimental conditions. Traditional clustering approaches, like for instance k-means algorithm, however, have proved to perform well only with a small set of expression data. With large datasets, composed of thousands of genes and hundreds of experimental conditions, clustering techniques are not able to correct identify the gene clusters, because they consider the expression profiles under all conditions at the same time. Many regulation mechanisms, indeed, involve only a set of genes and a limited set of experimental conditions $[28,29]$. For this reason, in recent years biclustering algorithms have been developed for the study of gene expression data [30]. A bicluster is defined as a group of genes showing a similar regulation behaviour over a subset of experimental conditions. Moreover biclustering allows to obtain overlapping biclusters, in which a gene can be involved in different regulation patterns according to the groups of considered conditions. The biclustering approach can contribute to interpret the big amount of RNA-seq data in an easier, faster and specific way. Moreover, as reported by literature, miRNAs are deregulated in breast cancer as well as in other hematopoietic and solid tumours $[2,6,14,19,20,23]$. Clustering those molecules according to subclass of tumours by histological and molecular point of view can help to better define a potential role of miRNA as prognostic, diagnostic and therapeutic markers.

The use of biclustering algorithm in order to correlate miRNA profiles to specific tumour features was supported by a recent work [31]. It showed the use of a biclustering approach to detect breast cancer subgroups with common clinical features. The proposed approach can be a new useful method to analyse and stratify treatment and predict prognosis, with respect to not fixed sets of genomic biomarkers.

The present work, that is an extended version of [32], will take into account miRNA expression profiles in breast cancer samples and healthy controls, in order to identify some patterns of deregulated miRNA in human breast cancer and to give a contribution in the understanding of the mechanism involved in the regulation of this type of cancer. 


\section{Methods}

Biclustering is a computational procedure that separates homogeneous sub-matrices inside a matrix composed of objects vs features. In our study the data matrix is constituted by samples vs miRNAs. The biclustering procedure selects simultaneously a subset of features that characterizes a subset of samples, on the basis of a "local similarity" criterion. On the contrary, traditional clustering groups together objects that are similar with respect to the whole set of features, not to a subset of features [30]. It is also clear that a proper biclustering technique is also different from a two-steps clustering that acts first on rows and then on columns of the data matrix, such as the double hierarchical clustering implemented in [33]. Considering our study, biclustering will find a subset of miRNAs that are differentially expressed with regards to a subset of samples. Among the available biclustering tools, we consider the Iterative Signature Algorithm (ISA) [34].

Figure 1 shows the whole pipeline used in this work. The input is a matrix of miRNA expression profiles, preprocessed using a standard distribution normalization, as recommended by ISA's authors. Then ISA algorithm is executed and the resulting biclustering (miRNA clusters vs patients) is represented with a heatmap visualization. Finally, there is the three steps validation of each bicluster, using the MetaMirClust online repository [35], the analysis of literature collected in UCSC Genome browser [36] and the consensus of our results by means of another biclustering technique, the SAMBA algorithm [37].

\section{Iterative signature algorithm}

According to recent surveys [38,39] that compare several biclustering algorithms on gene expression datasets, the ISA algorithm [34] is one of the most effective. Given a gene expression matrix, ISA algorithm discovers simultaneously sets of coregulated genes and the corresponding sets of experimental conditions. These biclusters are called transcription modules by ISA's authors. In our approach, the set of genes are represented by a set of miRNAs and the experimental conditions can be identified with breast cancer samples and healthy tissues.

Considering a gene expression matrix $E$ of size $m \times n$ (with $m=$ number of genes and $n=$ number of samples), the ISA algorithm works in the following way. First of all, it is created a random sparse $0 / 1$ vector of size $m$, called seed vector $r_{0}$. The elements of $r_{0}$ are used to select a subset of the genes (rows). $E$ is then transposed $\left(E^{\prime}\right)$, it is multiplied by $r_{0}$, and the result is thresholded, obtaining as result the vector $c_{0}$ of size $n$. This threshold is obtained computing the mean and standard deviations of the vector $c_{0}$, and considering only the elements that have a standard deviation from the mean higher than the threshold. The vector $c_{0}$ is then used to select a subset of the input samples, considering only the columns of $E$ corresponding to the non-zero components of $c_{0}$. After that, $E$ is then multiplied by $c_{0}$, and the result is again thresholded in order to obtain the vector $r_{1}$ (of size $m$ ). This procedure is iteratively repeated until either $r_{i-1}$ and $r_{i}$, and $c_{i-1}$ and $c i$ are close enough, according to a convergence criteria

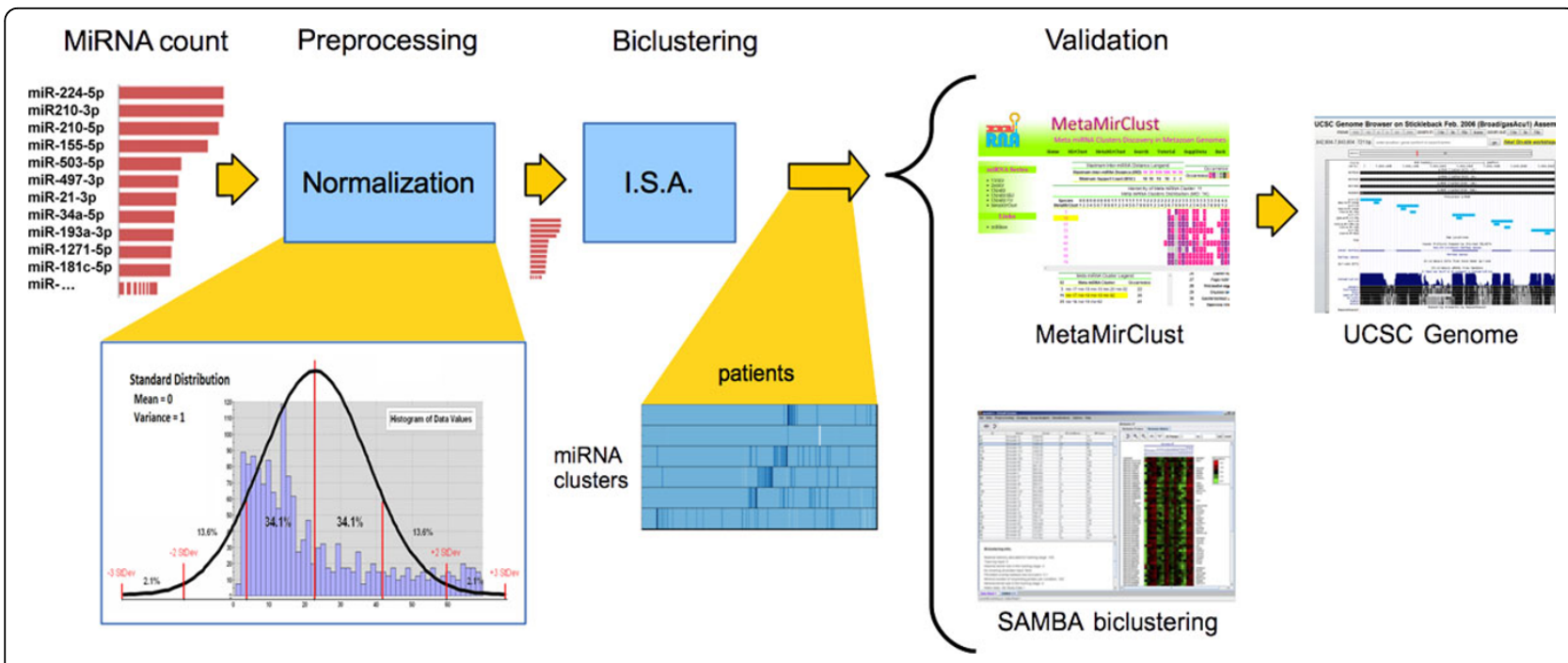

Figure 1 The proposed pipeline. Starting from miRNA count, we apply a normalization of data and perform a biclustering with ISA algorithm. The supervised evaluation exploits two online repositories, MetaMirClust and UCSC Genome browser, and validation by consensus with SAMBA algorithm. 
defined in terms of Pearson correlation. The final vectors $r_{\text {final }}$ and $c_{\text {final }}$ are used to extract a subset of the genes and samples, composing the biclusters, from the expression matrix $E$. Genes are selected considering the rows of $E$ corresponding to the the non-zero components of $r_{\text {final }}$; samples are selected considering the columns of $E$ corresponding to the the non-zero components of $c_{\text {final }}$. Therefore the two most important parameters of ISA algorithm are the two thresholds: $t h_{r}$ for the matrix rows (representing the genes), $t h_{c}$ for the matrix columns (associated to the samples). The threshold values affect the composition of the biclusters: for example, if the row (gene) threshold is high, then the biclusters will have very similar genes. Lower threshold values, in turn, will provide bigger biclusters with less similar genes. The same behaviour applies when considering the column (sample) threshold. Moreover the biclusters are "soft", meaning that they allow overlapping among genes and/or samples, and, according to the threshold values, it is possible that groups of genes and samples do not belong to any bicluster. It is also possible to obtain a bicluster that correlates a single gene with a group of patients and vice versa. As the authors state, ISA algorithm has the best performance processing normalized input data, i.e the input matrix have zero mean and standard deviation equal to one. In addition to the biclusters, ISA algorithm also provides as output two numeric matrices: one for the row (gene) components of the bicluster, and another one for the column (sample) components of the bicluster. Considering for example the resulting matrix for the genes, each column corresponds to a bicluster. The rows with a non-zero value belong to the bicluster related to that column. These matrices have values ranging from -1 and +1 and they express the association degree of a gene, or sample, to that bicluster. If two genes belong to the same bicluster with the same sign, they are correlated, otherwise they are anti-correlated. In the following discussions, for sake of simplicity, we take into account the output matrix having samples as rows, and the miRNA biclusters as column. Since the biclusters are unique, the same conclusions hold when considering miRNA vs sample biclusters.

In our work, we used the ISA implementation provided by the $\mathrm{R}$ package isa2 [40].

\section{Validation technique}

We adopted two different validation criteria: 1) semisupervised evaluation criterion, based on scientific literature; 2) consensus with another biclustering tool, the SAMBA algorithm [37], that has proven to discover relevant subsets of genes and samples from gene expression data [41]. Indeed, since we cannot perform in vitro experiments, we referred to: 1) an external repositories providing informations about different groups of miRNAs in human species; 2) a different biclustering algorithm to compare our results with.

In particular, we investigated over MetaMirClust database [35] and UCSC Genome browser [36]. The former is a repository of miRNAs in animalia kingdom that provides a useful bioinformatics resource to facilitate the advanced interrogations on the composition of miRNA clusters. The latter is a genome browser containing a collection of annotation for several genomic regions and, for each annotation, it reports its description, details about its construction, methods used in the experimentation, validation, credits, and references. Exploiting these two services, we could compare miRNA clusters obtained by the proposed technique with those contained in MetaMirClust and then we found for each bicluster all available literature references in UCSC Genome browser. Finally, we got information from references, in order to examine how they are related to differential expression of miRNAs in breast cancer. Our biclustering results were further validated using the SAMBA biclustering algorithm by checking if it is able to discover the same biclusters obtained through the ISA algorithm.

\section{Results and discussion}

In this section, we introduce the experimental dataset and then we discuss the biclustering results obtained according to the proposed pipeline, introduced in the Section Methods.

\section{Experimental dataset}

We used a breast cancer dataset composed of a large cohort of patients. RNA-seq data were collected by Farazi et al ([33]) and uploaded in Sequence Reads Archive data bank (SRA, http://www.ncbi.nlm.nih.gov/Traces/ sra/sra.cgi). The miRNA expression profiles were downloaded from Gene Expression Omnibus (GEO) with accession number GSE28884. The dataset is composed of 169 tumour samples, 10 healthy tissues, 6 cell lines (MCF7, MCF10A, HCC38, BT474, MDA-MB134, and ZR-751), for a total of 185 samples. We wanted, indeed, to test the efficacy of the ISA biclustering algorithm on a real dataset, not altered by a guided choice of the samples, and on a real life problem as the breast cancer analysis. Another parameter that we took into account was the partition of samples according to the clinical and histo-pathological features, molecular sub-type and the presence of one or more immune-histo-chemical (IHC) markers (ER, PR, Her2 status). Our aim is in fact to discover relationships between specific patterns of patients and specific groups of miRNA.

Our biclustering approach allowed us to evaluate miRNA deregulation in the context of miRNA abundance, and tissue heterogeneity, important elements in 
identifying miRNAs that would be useful prognostic and diagnostic markers.

Figure 2 shows the dataset composition. In order to verify if there could be some potential link between miRNA biclusters and some specific tumour characteristic, we considered three features (the list of patient features was provided by Farazi et al. [33]): a) presence or absence of IHC markers; b) clinical and histo-pathological features; c) molecular sub-type. For the IHC markers, point (a), it was considered the presence or absence in a tumour sample of Estrogen Receptor (ER), Progesteron Receptor (PR), or the ERB2/Her2 protein. As it can be seen the majority of patients are triple negative (46\%). From a molecular point of view these patients could be relevant to deeper understand the biology of these tumours and potentially to give answers in terms of pharmacological treatments. Regarding the clinical and histopathological features, point (b), patients are divided in IDC (Invasive Ductal) (68\%), DCIS (Ductal carcinoma In Situ) (9\%), Normal samples (healthy controls) (6\%), Metaplastic (5\%), Cell lines (3\%) and others (atypical medullary, apocrine, adenoid, mucinous A). It can be noticed that also this feature is non-homogeneous as the most part of analysed samples are IDC, compared to all the other tumour samples and healthy controls. The third characteristic, point (c) that we considered was the molecular sub-type, shared in basal (42\%), Luminal A (13\%), Her2 (12\%), Normal (11\%), Luminal B (3\%) and $19 \%$ of remaining samples were NA, because we did not have information regarding their molecular sub-type.

Regarding the miRNA profiles, we set the threshold for sample comparison to 5,000 total miRNA sequence reads per replicate merged library, as reported in [33], identifying 231 miRNA. miRNA profiles of a sample have been presented as relative percent (\%) miRNA read frequencies (rf) by dividing miRNA read counts by total
miRNA reads per library. Furthermore, miRNA profiles have been condensed by assigning individual miRNA to sequence families (denoted by $s f-m i R$ ). This approach reduces the complexity of the data. The sequence families are most informative for characterization of seed-sequence-dependent miRNA-target regulation. We considered the miRNA families as identified in the work of Farazi et al. [33] and provided in the Supplementary Table S4 available from the supplementary material of their work.

\section{Experimental results}

We aimed to evaluate the differential expression of miRNAs in breast cancer samples versus healthy tissues. Figure 3 shows the results obtained by the ISA algorithm. Membership values, ranging from -1 to +1 , are showed for every patient and each miRNA bicluster, as produced by the ISA algorithm. Starting from the heatmap visualisation, we considered the patients belonging to the miRNA bicluster with the highest membership value. The differential expression analysis evidenced 12 different miRNA clusters. Each of these was associated to a specific cluster of patients. Figure 4 summarizes the composition of each bicluster, their references in scientific literature and if they were also discovered by the SAMBA algorithm, as explained in Section Validation Technique. Since the analysis of all the biclustering results obtained through SAMBA algorithm is not in the scope of this work, we provide them in the Supplementary Material, Additional file 1 and 2, for sake of completeness.

The two thresholds of the ISA algorithm were regulated in order to have a manageable (not too large) and meaningful number of biclusters. $t h_{r}$ was set equal to 3.5, $t h_{c}$ was set equal to 1.0. Other threshold values gave

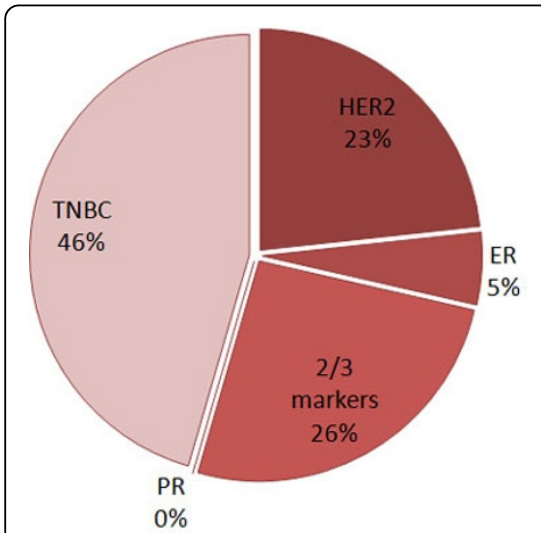

(a) IHC Markers

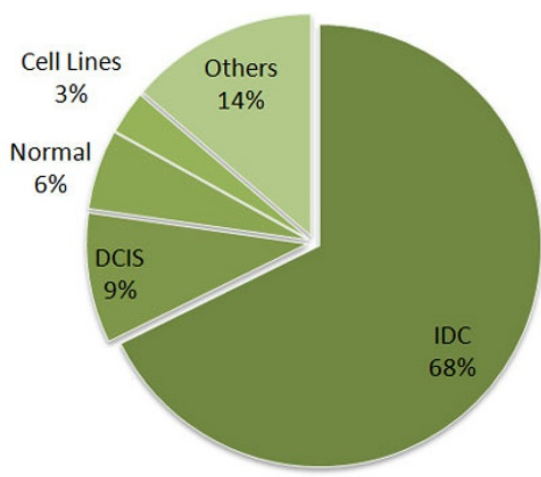

(b) Clinical and histopathological features

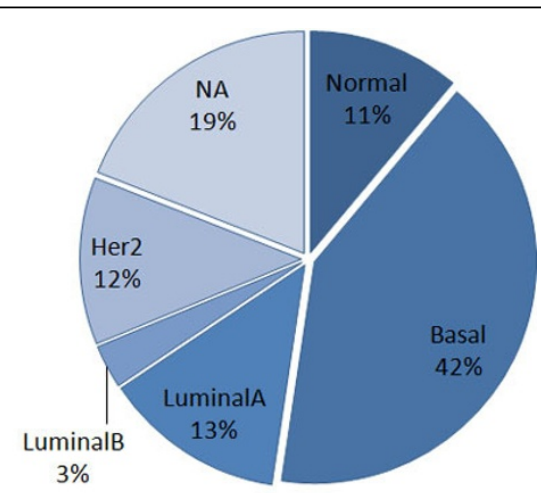

(c) Molecular sub-type

Figure 2 Dataset Composition. The real world dataset is composed by 185 samples. Each pie represents a different characteristic, where the percentage values give information about the composition of the dataset. 


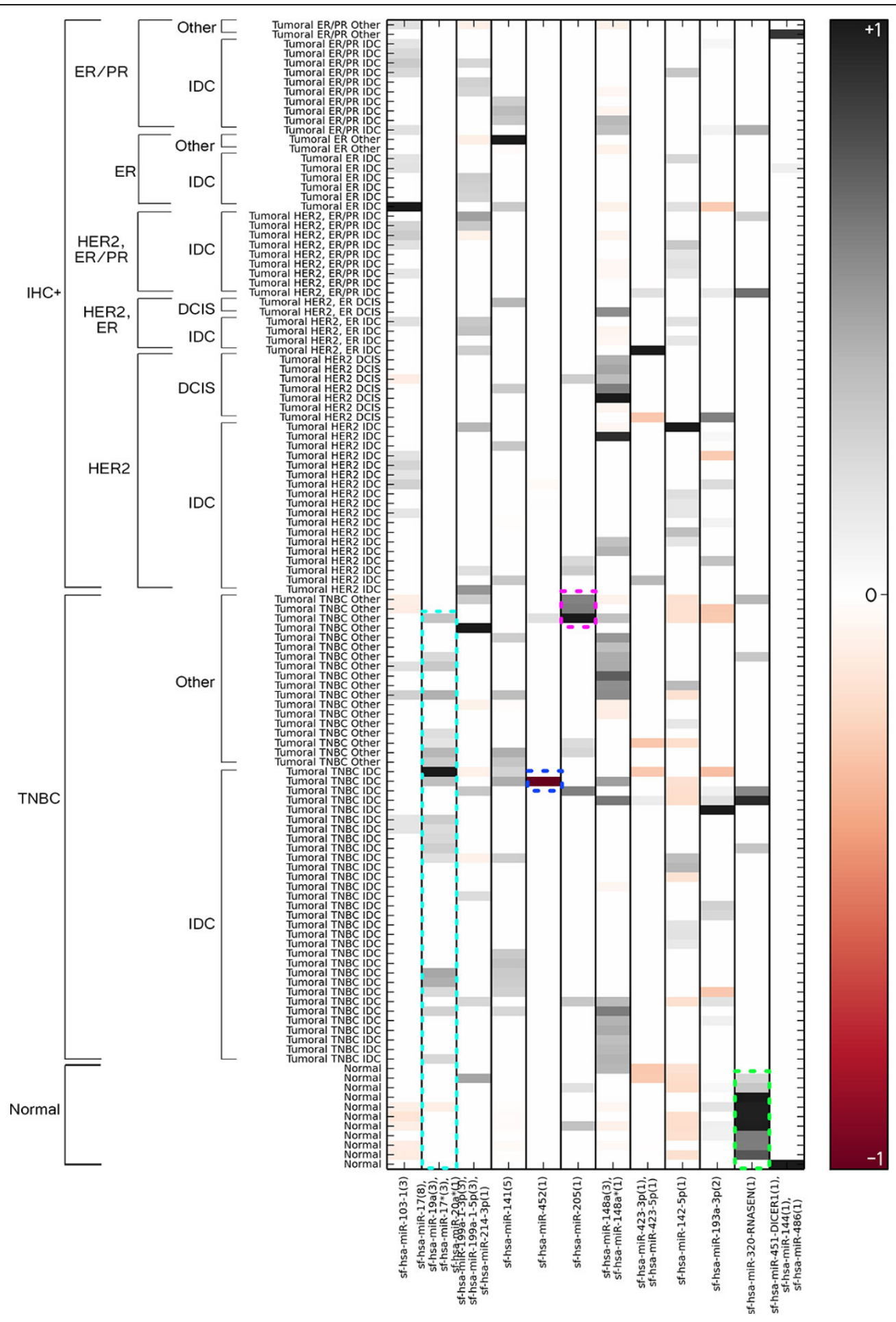

Figure 3 Bicluster visualization. The heatmap shows the membership of each patient with regards to the 12 miRNA biclusters. Only the patients belonging to at least one miRNA bicluster are reported. Membership ranges from -1 to +1 , with 0 meaning no membership. +1 and -1 indicate highest membership, but with opposite correlation degree. The heatmap indicates a strong relationship between some features of the dataset and specific subclass of miRNA. Indeed the expression patterns of miRNA family hsa-mir-17/19a/17*/20a* inside the azure rectangle shows an almost exclusive link with TNBC samples. The pink rectangle in miRNA-205 links this non coding RNA to a specific subclass of BC samples. The blue rectangle evidences an inverse correlation between the mir-452 and some IDC samples. The grey rectangle evidences the association to mir-320-RNASEN(1) with histological normal samples 


\begin{tabular}{|c|c|c|c|c|c|c|c|}
\hline \multicolumn{3}{|c|}{ Biclusters } & \multicolumn{3}{|c|}{ Sample Features } & \multirow{2}{*}{$\begin{array}{l}\text { Scientific } \\
\text { Literature } \\
\text { References }\end{array}$} & \multirow{2}{*}{$\begin{array}{l}\text { Related } \\
\text { SAMBA } \\
\text { Bicluster }\end{array}$} \\
\hline$\#$ & miRNA & sample & \# & Histological Type & IHC marker & & \\
\hline \multirow{3}{*}{1} & \multirow{3}{*}{ sf-hsa-miR-320-RNASEN(1) } & \multirow{3}{*}{12} & \begin{tabular}{l|}
1 \\
1
\end{tabular} & IDC & $\begin{array}{l}\text { ER+/PR+ } \\
\text { her2+ }\end{array}$ & \multirow{3}{*}{ [33] } & \multirow{3}{*}{2} \\
\hline & & & $\frac{1}{8}$ & NORMAL & $\begin{array}{l}\text { her2+ } \\
\text { NORMAL }\end{array}$ & & \\
\hline & & & 2 & IDC & TNBC & & \\
\hline \multirow{11}{*}{2} & \multirow{11}{*}{$\begin{array}{c}\text { sf-hsa-miR-148a(3) } \\
\text { sf-hsa-miR-148a*(1) }\end{array}$} & \multirow{11}{*}{31} & 1 & & ER+ & \multirow{11}{*}[60-62]{} & \multirow{11}{*}{-} \\
\hline & & & 1 & & TNBC & & \\
\hline & & & 1 & cell line & TNBC & & \\
\hline & & & 1 & TDC & $\mathrm{ER}+/ \mathrm{PR}+$ & & \\
\hline & & & 7 & DCIS & her2+ & & \\
\hline & & & 3 & TDC & her $2+$ & & \\
\hline & & & 1 & NORMAL & NORMAL & & \\
\hline & & & 2 & Apocrin & & & \\
\hline & & & 4 & Atypical Medullary & & & \\
\hline & & & 8 & TDC & & & \\
\hline & & & 2 & Metaplastic & TNBC & & \\
\hline \multirow[b]{2}{*}{3} & \multirow{2}{*}{$\begin{array}{l}\text { sf-hsa-miR-17(8) } \\
\text { sf-hsa-miR-19a(3) } \\
\text { sf-hsa-miR-17*(3) } \\
\text { sf-hsa-miR-20a*(1) }\end{array}$} & \multirow[b]{2}{*}{7} & 6 & IDC- basal & \multirow[b]{2}{*}{ TNBC } & \multirow[b]{2}{*}{$\begin{array}{c}{[33]} \\
{[44,45]}\end{array}$} & \\
\hline & & & 1 & Metaplastic-basal & & & - \\
\hline & & & 1 & & ER+ & & \\
\hline & & & 1 & & ER+/PR+ & & \\
\hline 4 & sf-hsa-miR-142-5p(1) & 16 & 7 & IDC & her2+ & [33] & 10 \\
\hline & & & 1 & Atypical Medullary & TNBC & & \\
\hline & & & 6 & TDC & TNBC & & \\
\hline & & & 3 & & ER+ & & \\
\hline & & & 2 & & $\mathrm{ER}+/ \mathrm{PR}+$ & & \\
\hline & sf-hsa-miR-199a-1-3p(3) & & 6 & IDC & her2+ & & \\
\hline 5 & sf-hsa-miR-199a-1-5p(3) & 14 & 1 & NORMAL & NORMAL & {$[42,43]$} & 9 \\
\hline & & & 1 & TDC & & & \\
\hline & & & 1 & Metaplastic & TNBC & & \\
\hline & & & 1 & TDC & her2+ & & \\
\hline & & & 1 & Adenoid & TNBC & & \\
\hline 6 & sf-hsa-miR-205(1) & 4 & 1 & IDC & IIVBC & {$[46,47]$} & - \\
\hline & & & 1 & Metaplastic & TNBC & & \\
\hline 7 & sf-hsa-miR-423-3p(1) & 3 & 2 & TDC & her2+ & [33] & 6 \\
\hline 1 & sf-hsa-miR-423-5p(1) & 3 & 1 & Adenoid & TNBC & {$[48-50]$} & \\
\hline & & & 1 & & $\mathrm{ER}+/ \mathrm{PR}+$ & & \\
\hline & & & 1 & cell line & her2+ & & \\
\hline 8 & sf-hsa-miR-103-1(3) & 12 & 2 & & ER+ & [51] & 4 \\
\hline & & & 3 & & $\mathrm{ER}+/ \mathrm{PR}+$ & & \\
\hline & & & 5 & IDC & her2t & & \\
\hline & & & 1 & cell line & ER+ & & \\
\hline & & & 2 & TDC & $\mathrm{ER}+/ \mathrm{PR}+$ & {$[54-56]$} & \\
\hline 9 & sf-hsa-miR-141(5) & 10 & 2 & DCIS & her2+ & {$[58,59]$} & - \\
\hline & & & 5 & TDC & TNBC & & \\
\hline 10 & sf-hsa-miR-452(1) & 1 & 1 & IDC & TNBC & {$[52]$} & - \\
\hline & & & 1 & Atypical Medullary & & & \\
\hline & & & 1 & DCIS & & & \\
\hline 11 & sf-hsa-miR-193a-3p(2) & 7 & 2 & & her2+ & [53] & - \\
\hline & & & 3 & IDC & TNBC & & \\
\hline & & & 1 & mucinous $A$ & $\mathrm{ER}+/ \mathrm{PR}+$ & & \\
\hline 12 & $\begin{array}{l}\text { st-hsa-miR-45I-DICERI(1) } \\
\text { sf-hsa-miR-144(1) }\end{array}$ & 2 & & & & {$[63,64]$} & 1 \\
\hline & sf-hsa-miR-486(1) & & 1 & NORMAL & NORMAL & & \\
\hline
\end{tabular}

Figure 4 Table of biclustering results. It reports, for each obtained bicluster, the group of miRNAs and the number of patients. In addition, for each patient in bicluster, some main characteristics are reported. The last two columns contain references provided by literature works and the number of the related bicluster, if any, discovered by the SAMBA algorithm. All the biclusters obtained with SAMBA algorithm are provided in the Supplementary material, Additional file 1 and 2. See caption of Figure 3 for details about red, blue, azure and green rectangles. 
few clusters but with many elements, or many clusters with few elements. Considering the random initialization of ISA algorithm we ran it ten times with different initialization values but with the same thresholds, in order to assess the stability of the 12 biclusters. Each time we obtained the same 12 biclusters, although with a different sorting. As it is evident in Figure 4, 7 out of 12 biclusters are composed of one sequence family. Unlikely traditional clustering methods, where a cluster with only one element has often very low importance, a bicluster that correlates a single element with a group of features give us a lot of information.

As it will be discussed in the rest of this section, indeed, biclusters composed of one single miRNA family linked to a group of samples, enable us to associate a particular miRNA family to a set of sample tissues with well defined immuno-histochemical and histological features. This characteristic of the algorithm allows in a "one step" process to obtain results that are normally produced with more passages with a classical hierarchical clustering process.

Our biclustering procedure highlighted many results from the original work of Farazi et al [33], it also produced other results that are confirmed by other works in the literature, and some new results that need more investigation. First of all we comment the results in accordance with the original Farazi work.

The first step of our analysis was to check if the healthy tissues were univocally associated to the same sub-class of miRNA. The data showed an exclusive association between these samples and the miRNA cluster sf-hsa-miR-320-RNASEN (green rectangle on Figure 3 and Figure 4). The obtained data were confirmed by Farazi et al. [33] and by the SAMBA algorithm (bicluster 2 , available in the supplementary material, Additional file 1). Moreover, as it will be argued, the algorithm showed the association between specific sub-class of tumour samples having the same immuno-histo-chemical (IHC) and/or histological features and miRNA clusters, as can be evidenced for "atypical medullary" and DCIS samples that are linked to sf-hsa-miR-148a* and sf-hsa-miR-148a clusters. The bicluster evidenced also an over-expression of sf-hsa-miR-142-5p in Her2+/IDC samples compared to other classes of tumour samples, as DCIS or TNBC, and compared to healthy controls. These results were in accordance with the evidence reported by Farazi et al [33]. SAMBA biclustering showed an association between this miRNA bicluster, $\mathrm{ER}+/ \mathrm{PR}+/ \mathrm{IDC}$, and HER2+/IDC samples, confirming our results. Indeed there is an over-expression of this group of microRNA with regards to these classes of samples. Moreover the algorithm reported a downexpression of TNBC samples for this miRNA cluster (supplementary material, Additional file 1 bicluster 10).
Some IDC samples have shown an association to miRNA sf-hsa-miR-199a-1-3p, sf-hsa-miR199a-1-5p e sf-hsa-miR-214-3p clusters, that were over-expressed. Schwarzenbach et al. and Shatseva et al. confirmed our data clustering, showing increased levels of mir-214 in serum of $\mathrm{BC}$ patients compared to healthy controls $[42,43]$. The analysis with SAMBA algorithm reported also an over-expression for ER+/PR+/IDC, Her2+/IDC samples, confirming our results; it also reported a down-expression for some TNBC samples not evidenced with ISA biclustering (supplementary material, Additional file 1 bicluster 9 ).

Triple Negative Breast Cancer samples (TNBC) are a distinct group of tumour samples characterized from distinct histological and IHC features, as they lack for the presence of estrogen receptor (ER), progesterone receptor (PR), and Her2 protein receptors. They are associated to a specific pattern of miRNA, that is disregulated: sf-hsa-miR-17, sf-hsa-miR-19a, sf-hsa-miR-17*, and sf-hsa-miR-20a* (azure rectangle on Figure 3 and Figure 4). These are oncogenes and their targets are "tumour suppressors" of the cell cycle. Many scientific works report an over-expression of this cluster of miRNA in different types of solid tumours and hematopoietic diseases $[33,44]$. Especially, this association involves a specific sub-class of TNBC that is the "basal like subtype", as reported also by De Rinaldis et al. [45]. This evidence suggests the tendency of specific miRNA that are "sub-type specific" to influence the expression levels of their targets. The identification of a cluster of miRNA specifically linked to the TNBC sub-class of samples is very important because nowadays there are not targeted therapies for this $\mathrm{BC}$ sub-class. The discovery of miRNAs associated with TNBC could mean to find a less invasive strategy of intervention. Differently from our results, SAMBA biclustering reported a downexpression for miR-17* and miR-20* in TNBC samples (supplementary material, Additional file 1 bicluster 7).

In literature, data about the role of miRNA-205 are conflicting: our biclustering approach highlighted an over-expression of this miRNA in some IDC, adenoid and metaplastic samples (pink rectangle on Figure 3 and Figure 4). Interestingly, a closer look to the IHC features evidenced that this specific class of miRNA strongly associates to TNBC samples, as the values reported by the algorithm are almost equal to 1 in absolute value. This data was in accordance with the result obtained by Farazi et al. [33]. No association was found for this bicluster with SAMBA analysis. Other miRNA profiling has shown increased levels of this miRNA-205 in BC samples without vascular invasion compared to those with vascular invasion. Moreover miR-205 has been shown to be down-regulated in $\mathrm{BC}$ tumour samples and BC cellular lines $[46,47]$. 
miR-423-3p and miR-423-5p are the mature transcripts of miR-423. Many scientific works reported that their expression was altered in different forms of cancer type [33,48-50]. Farazi et al. reported that these two the mature forms of miR-423 were highly expressed in ductal infiltrating $\mathrm{BC}$ that subsequently give metastasis, underlying the prognostic independent role of the disease [33]. Our biclustering analysis evidenced a distinct group of IDC patients where miR-423 was overexpressed. SAMBA biclustering showed an association with only miR-423-5p and some distinct groups of samples: an over-expression was associated to Her2+/IDC samples, and a down-expression was found with ER $+/ \mathrm{PR}+/$ mucinous A samples (supplementary material, Additional file 1 bicluster 6).

The proposed biclustering procedure gave some new results discussed below; many of these findings are confirmed by other papers in the literature. As general observation we found that most of the results are related to over-expressed miRNAs, with only one bicluster (row 10 in Figure 4) involving down-regulated miRNAs.

The differential expression analysis of sf-hsa-miR-1031 showed an over-expression in IDC cluster samples, positive for at least one of three IHC marker (PR/ER/ Her2). These results have been confirmed by SAMBA biclustering (supplementary material, Additional file 1 bicluster 4). A recent work of Wang X. evidenced a potential role of this microRNA as serum biomarker in $\mathrm{BC}$ patients. Moreover its expression correlates with an advanced state of the disease $(\mathrm{P}<0.05)$ and with linfonodal metastasis $(\mathrm{P}<0.05)$ [51].

The sf-hsa-miR-452 is involved in different processes as cell-cell interaction, cell growth and proliferation, inflammatory response and apoptotic processes. The biclustering algorithm showed a strong down-expression of this miRNA family (blue rectangle on Figure 3 and Figure 4). Interestingly Van Schooneveld et al. [52] referred an over-expression in healthy patients compared to $\mathrm{BC}$ patients, and they evidenced a positive association $(\mathrm{P}<0.05)$ between expression levels of miR452 in serum of patients and the number of methylated genes in plasma. The experimental evidences showed a strong over-expression of miR-452 in healthy controls compared to tumour samples, and because of the evidence of the stability of this miRNA in plasma, the authors speculated a potential role as biomarker for the detection of $\mathrm{BC}$ in human plasma. SAMBA biclustering reported no association for this miRNA.

The biclustering analysis showed an over-expression of sf-hsa-miR-193a-3p in IDC sub-class of patients. An over-expression of this miRNA in tumour samples was previously evidenced in other works as reported by Fung Lin Yong [53]. No association was found with SAMBA algorithm.
Our algorithm produced also some results that are often discordant from literature: miR-141 and miR-200c belong to a miRNA family of important regulator of cellular transition from epithelial to mesenchymal (EMT) [54-56]. Moreover, miR-200c has been shown to play a role in disregulation of normal expression patterns of different cancer types [18,57]. Our analysis evidenced an over-expression of sf-hsa-miR-141 in tumour samples compared to healthy controls. Most of them are IDC, and Her2 negatives. In literature data are contrasting $[58,59]$. Moreover SAMBA biclustering reported no results for this miRNA bicluster.

As previously said, sf-hsa-miR-148a* and sf-hsa-miR148a miRNA clusters were linked by the algorithm analysis to "atypical medullary" and DCIS samples. Also these data differ from literature: scientific works conducted on different cancer types showed a down-regulation in tumour samples compared with healthy controls [60-62]. No association was found with SAMBA biclustering.

A new association is reported about the miRNA bicluster composed of sf-hsa-miR-451-DICER1, sf-hsamiR-144, sf-hsa-miR-486: our biclustering showed an over-expression of this bicluster in a sub-class of IDC/ her2 positive patients. In literature there are no data regarding an association about this last miRNA bicluster and breast cancer but there are more evidence about an over-expression of miRNA 451 in BC and other tumour samples $[63,64]$. Instead in [33] it is reported that miR144 and miR-451 clustered together but were abundant in normal breast and reduced in IDC. Interestingly SAMBA algorithm also reported the association among miR-451-DICER1, miR-144 and miR-486. For this miRNA bicluster was evidenced an over-expression in $\mathrm{ER}+/ \mathrm{PR}+$ samples (supplementary material, Additional file 1 cluster 1 ).

\section{Conclusions}

In this paper we used a biclustering approach, by means of the ISA algorithm, in order to analyse the differential expression of miRNA in breast cancer samples. Thanks to its features, the biclustering method allows to cluster subset of patients with subset of miRNAs, exploiting this way miRNA biclusters under different samples conditions. Our analysis highlighted 12 miRNA biclusters, each of them involving different types of tumour samples, characterized by immuno-histo-chemical, histopathological and molecular sub-type features. Biclusters were validated in the current scientific literature with the support of the MetaMirClust and UCSC Genome Browser online tools. Further validation was provided by the consensus with another biclustering tool, the SAMBA algorithm. The proposed biclustering methodology has proved a valid instrument for the study of miRNA expression profiles, with the possibility to identify biclusters that can 
provide novel relationships among groups of miRNAs and patient conditions. Of course, discordant or new results need to be deeply investigated.

\section{Additional material}

Additional file 1: SAMBA biclustering results. Excel file summarizing the whole biclustering results obtained with SAMBA algorithm. Each sheet of the file reports the composition, in terms of miRNA and sample, of each bicluster.

Additional file 2: Bicluster heathmaps. An archive containing the heatmaps of each bicluster obtained with SAMBA algorithm.

\section{Competing interests}

The authors declare that they have no competing interests.

\section{Authors' contributions}

AF: project conception, implementation, discussions, assessment, writing. MLR: project conception, implementation, discussions, assessment, writing. LLP: project conception, experimental tests, discussions, assessment, writing RR: project conception, discussions, assessment, writing. AU: project conception, discussions, assessment, writing, funding. All authors read and approved the final manuscript.

\section{Declarations}

The publication costs for this article were funded by the project PO-FESR SICILIA 4.1.1.1 - "MIRCO - MicroRna in Clinica Oncologica".

This article has been published as part of BMC Bioinformatics Volume 16 Supplement 4, 2015: Selected articles from the 9th IAPR conference on Pattern Recognition in Bioinformatics. The full contents of the supplement are available online at http://www.biomedcentral.com/bmcbioinformatics/ supplements/16/S4.

\section{Authors' details}

'ICAR-CNR, National Research Council of Italy, viale delle Scienze, Ed.11, 90145 Palermo, Italy. ${ }^{2}$ ICAR-CNR, National Research Council of Italy, via P. Castellino 111, 80131 Napoli, Italy.

Published: 23 February 2015

\section{References}

1. Du T, Zamore PD: microPrimer: the biogenesis and function of microRNA. Development (Cambridge, England) 2005, 132(21):4645-52.

2. Schee K, Fodstad Oy, Flatmark K: MicroRNAs as biomarkers in colorectal cancer. The American journal of pathology 2010, 177(4):1592-9.

3. He L, Hannon GJ: MicroRNAs: small RNAs with a big role in gene regulation. Nature reviews. Genetics 2004, 5(7):522-31.

4. Kim VN: MicroRNA biogenesis: coordinated cropping and dicing. Nature reviews. Molecular cell biology 2005, 6(5):376-85.

5. Petersen CP, Bordeleau M-E, Pelletier J, Sharp PA: Short RNAs repress translation after initiation in mammalian cells. Molecular cell 2006 21(4):533-42.

6. Lu J, Getz G, Miska EA, Alvarez-Saavedra E, Lamb J, Peck D, SweetCordero A, Ebert BL, Mak RH, Ferrando AA, Downing JR, Jacks T, Horvitz HR, Golub TR: MicroRNA expression profiles classify human cancers. Nature 2005, 435(7043):834-8,

7. Esquela-Kerscher A, Slack FJ: Oncomirs - microRNAs with a role in cancer. Nature reviews. Cancer 2006, 6(4):259-69.

8. Bartel DP: MicroRNAs: genomics, biogenesis, mechanism, and function. Cell 2004, 116(2):281-97.

9. Vasudevan S, Tong Y, Steitz JA: Switching from repression to activation: microRNAs can up-regulate translation. Science (New York, N.Y.) 2007, 318(5858):1931-4.

10. Mitchell PS, Parkin RK, Kroh EM, Fritz BR, Wyman SK, PogosovaAgadjanyan EL, Peterson A, Noteboom J, O'Briant KC, Allen A, Lin DW Urban N, Drescher CW, Knudsen BS, Stirewalt DL, Gentleman R, Vessella RL,
Nelson PS, Martin DB, Tewari M: Circulating microRNAs as stable bloodbased markers for cancer detection. Proceedings of the National Academy of Sciences of the United States of America 2008, 105(30):10513-8.

11. Lewis $B P$, Burge $C B$, Bartel DP: Conserved seed pairing, often flanked by adenosines, indicates that thousands of human genes are microRNA targets. Cell 2005, 120(1):15-20

12. Marson A, Levine SS, Cole MF, Frampton GM, Brambrink T, Johnstone $\mathrm{S}$, Guenther MG, Johnston WK, Wernig M, Newman J, Calabrese JM, Dennis LM, Volkert TL, Gupta S, Love J, Hannett N, Sharp PA, Bartel DP, Jaenisch R, Young RA: Connecting microRNA genes to the core transcriptional regulatory circuitry of embryonic stem cells. Cell 2008, 134(3):521-33.

13. Brennecke J, Stark A, Russell RB, Cohen SM: Principles of microRNA-target recognition. PLoS biology 2005, 3(3):85

14. Calin GA, Dumitru CD, Shimizu M, Bichi R, Zupo S, Noch E, Aldler $H_{\text {, }}$ Rattan S, Keating M, Rai K, Rassenti L, Kipps T, Negrini M, Bullrich F, Croce CM: Frequent deletions and down-regulation of micro-RNA genes miR15 and miR16 at 13q14 in chronic lymphocytic leukemia. Proceedings of the National Academy of Sciences of the United States of America 2002, 99(24):15524-9.

15. Fujita Y, Kojima K, Hamada N, Ohhashi R, Akao Y, Nozawa Y, Deguchi T, Ito M: Effects of miR-34a on cell growth and chemoresistance in prostate cancer $\mathrm{PC} 3$ cells. Biochemical and biophysical research communications 2008, 377(1):114-9.

16. Tazawa H, Tsuchiya N, Izumiya M, Nakagama H: Tumor-suppressive miR34a induces senescence-like growth arrest through modulation of the E2F pathway in human colon cancer cells. Proceedings of the National Academy of Sciences of the United States of America 2007, 104(39):15472-7.

17. Schetter AJ, Leung SY, Sohn JJ, Zanetti KA, Bowman ED, Yanaihara N, Yuen ST, Chan TL, Liu C-G, Calin GA, Croce CM, Harris CC: MicroRNA expression profiles associated with prognosis and therapeutic outcome in colon adenocarcinoma. JAMA : the journal of the American Medical Association 2008, 299(4):425-36

18. Volinia S, Calin GA, Liu C-G, Ambs S, Cimmino A, Petrocca F, Visone R, lorio M, Roldo C, Ferracin M, Prueitt RL, Yanaihara N, Lanza G, Scarpa A, Vecchione A, Negrini M, Harris CC, Croce CM: A microRNA expression signature of human solid tumors defines cancer gene targets. Proceedings of the National Academy of Sciences of the United States of America 2006, 103(7):2257-61.

19. O'Day E, Lal A: MicroRNAs and their target gene networks in breast cancer. Breast cancer research : BCR 2010, 12(2):201.

20. Foekens JA, Sieuwerts AM, Smid M, Look MP, de Weerd V, Boersma AWM, Klijn JGM, Wiemer EAC, Martens JWM: Four miRNAs associated with aggressiveness of lymph node-negative, estrogen receptor-positive human breast cancer. Proceedings of the National Academy of Sciences of the United States of America 2008, 105(35):13021-6.

21. Camps C, Buffa FM, Colella S, Moore J, Sotiriou C, Sheldon H, Harris AL, Gleadle JM, Ragoussis J: hsa-miR-210 Is induced by hypoxia and is an independent prognostic factor in breast cancer. Clinical cancer research an official journal of the American Association for Cancer Research 2008, 14(5):1340-8.

22. Ma $\mathrm{L}$, Teruya-Feldstein J, Weinberg RA: Tumour invasion and metastasis initiated by microRNA-10b in breast cancer. Nature 2007, 449(7163):682-8.

23. Li N, Fu H, Tie Y, Hu Z, Kong W, Wu Y, Zheng X: miR-34a inhibits migration and invasion by down-regulation of c-Met expression in human hepatocellular carcinoma cells. Cancer letters 2009, 275(1):44-53.

24. Marioni JC, Mason CE, Mane SM, Stephens M, Gilad Y: RNA-seq: an assessment of technical reproducibility and comparison with gene expression arrays. Genome research 2008, 18(9):1509-17.

25. Shalon D, Smith SJ, Brown PO: A DNA microarray system for analyzing complex DNA samples using two-color fluorescent probe hybridization. Genome research 1996, 6(7):639-45.

26. Schena M, Heller RA, Theriault TP, Konrad K, Lachenmeier E, Davis RW: Microarrays: biotechnology's discovery platform for functional genomics. Trends in biotechnology 1998, 16(7):301-6.

27. Wang Z, Gerstein M, Snyder M: RNA-Seq: a revolutionary tool for transcriptomics. Nature reviews. Genetics 2009, 10(1):57-63.

28. Ben-Dor A, Chor B, Karp R, Yakhini Z: Discovering local structure in gene expression data: the order-preserving submatrix problem. Journal of computational biology : a journal of computational molecular cell biology 2003, 10(3-4):373-84 
29. Ihmels J, Friedlander G, Bergmann S, Sarig O, Ziv Y, Barkai N: Revealing modular organization in the yeast transcriptional network. Nature genetics 2002, 31(4):370-7.

30. Madeira SC, Oliveira AL: Biclustering algorithms for biological data analysis: a survey. IEEE/ACM transactions on computational biology and bioinformatics 2004, 1(1):24-45.

31. Wang YK, Print CG, Crampin EJ: Biclustering reveals breast cancer tumour subgroups with common clinical features and improves prediction of disease recurrence. BMC genomics 2013, 14:102

32. Fiannaca A, La Rosa M, La Paglia L, Rizzo R, Urso A: Analysis of miRnA Expression Profiles in Breast Cancer Using Biclustering. In Pattern Recognition in Bioinformatics. Volume 8626. Lecture Notes in Computer Science, Springer, Berlin, Heidelberg; 2014:110-111.

33. Farazi TA, Horlings HM, Ten Hoeve JJ, Mihailovic A, Halfwerk $H$, Morozov $P$, Brown M, Hafner M, Reyal F, van Kouwenhove M, Kreike B, Sie D, Hovestadt V, Wessels LFA, van de Vijver MJ, Tuschl T: MicroRNA sequence and expression analysis in breast tumors by deep sequencing. Cancer research 2011, 71(13):4443-53

34. Bergmann S, Ihmels J, Barkai N: Iterative signature algorithm for the analysis of large-scale gene expression data. Physical Review E 2003, 67(3):031902.

35. Chan W-C, Ho M-R, Li S-C, Tsai K-W, Lai C-H, Hsu C-N, Lin W-C: MetaMirClust: discovery of miRNA cluster patterns using a data-mining approach. Genomics 2012, 100(3):141-8.

36. Fujita PA, Rhead B, Zweig AS, Hinrichs AS, Karolchik D, Cline MS, Goldman M, Barber GP, Clawson H, Coelho A, Diekhans M, Dreszer TR, Giardine BM, Harte RA, Hillman-Jackson J, Hsu F, Kirkup V, Kuhn RM, Learned K, Li CH, Meyer LR, Pohl A, Raney BJ, Rosenbloom KR, Smith KE, Haussler D, Kent WJ: The UCSC Genome Browser database: update 2011. Nucleic acids research 2011, , 39 Database: 876-82.

37. Tanay A, Sharan R, Shamir R: Discovering statistically significant biclusters in gene expression data. Bioinformatics 2002, 18(Suppl 1):136-44.

38. Li L, Guo Y, Wu W, Shi Y, Cheng J, Tao S: A comparison and evaluation of five biclustering algorithms by quantifying goodness of biclusters for gene expression data. BioData mining 2012, 5(1):8.

39. Eren K, Deveci $M$, Küçüktunç $O, C ̧$ atalyürek UV: A comparative analysis of biclustering algorithms for gene expression data. Briefings in bioinformatics 2013, 14(3):279-92.

40. Csárdi G, Kutalik Z, Bergmann S: Modular analysis of gene expression data with R. Bioinformatics (Oxford, England) 2010, 26(10):1376-7.

41. Oghabian A, Kilpinen S, Hautaniemi S, Czeizler E: Biclustering methods: biological relevance and application in gene expression analysis. PloS one 2014, 9(3):90801.

42. Schwarzenbach $H$, Milde-Langosch K, Steinbach B, Müller V, Pantel K: Diagnostic potential of PTEN-targeting miR-214 in the blood of breast cancer patients. Breast cancer research and treatment 2012, 134(3):933-41.

43. Shatseva T, Lee DY, Deng Z, Yang BB: MicroRNA miR-199a-3p regulates cell proliferation and survival by targeting caveolin-2. Journal of cell science 2011, 124(Pt 16):2826-36.

44. He L, Thomson JM, Hemann MT, Hernando-Monge E, Mu D, Goodson S, Powers S, Cordon-Cardo C, Lowe SW, Hannon GJ, Hammond SM: A microRNA polycistron as a potential human oncogene. Nature 2005, 435(7043):828-33.

45. de Rinaldis E, Gazinska P, Mera A, Modrusan Z, Fedorowicz GM, Burford B, Gillett C, Marra P, Grigoriadis A, Dornan D, Holmberg L, Pinder S, Tutt A: Integrated genomic analysis of triple-negative breast cancers reveals novel microRNAs associated with clinical and molecular phenotypes and sheds light on the pathways they control. BMC genomics 2013, 14:643.

46. Wu H, Zhu S, Mo Y-Y: Suppression of cell growth and invasion by miR205 in breast cancer. Cell research 2009, 19(4):439-48.

47. Iorio MV, Casalini P, Piovan C, Di Leva G, Merlo A, Triulzi T, Ménard S, Croce CM, Tagliabue E: microRNA-205 regulates HER3 in human breast cancer. Cancer research 2009, 69(6):2195-200.

48. Guled M, Lahti L, Lindholm PM, Salmenkivi K, Bagwan I, Nicholson AG, Knuutila S: CDKN2A, NF2, and JUN are dysregulated among other genes by miRNAs in malignant mesothelioma - A miRNA microarray analysis. Genes, chromosomes \& cancer 2009, 48(7):615-23.

49. Lenarduzzi M, Krushel T, Waldron L, Pintilie M, Shi W, Perez-Ordonez B, Jurisica I, O'Sullivan B, Waldron J, Gullane P, Cummings B, Liu F-F: Comprehensive MicroRNA profiling for head and neck squamous cell carcinomas. Clinical cancer research : an official journal of the American Association for Cancer Research 2010, 16(4):1129-39.

50. Lerman G, Avivi C, Mardoukh C, Barzilai A, Tessone A, Gradus B, Pavlotsky F, Barshack I, Polak-Charcon S, Orenstein A, Hornstein E, Sidi Y, Avni D: MiRNA expression in psoriatic skin: reciprocal regulation of hsa-miR-99a and IGF-1R. PloS one 2011, 6(6):20916.

51. Wang $X$, Wu $X$, Yan L, Shao J: Serum miR-103 as a potential diagnostic biomarker for breast cancer. Journal of Southern Medical University 2012, 32(5):631-4.

52. van Schooneveld E, Wouters MC, Van der Auwera I, Peeters DJ, Wildiers $H$, Van Dam PA, Vergote I, Vermeulen PB, Dirix LY, Van Laere SJ: Expression profiling of cancerous and normal breast tissues identifies microRNAs that are differentially expressed in serum from patients with (metastatic) breast cancer and healthy volunteers. Breast cancer research : BCR 2012, 14(1):34.

53. Yong FL, Law CW, Wang CW: Potentiality of a triple microRNA classifier: miR-193a-3p, miR-23a and miR-338-5p for early detection of colorectal cancer. BMC cancer 2013, 13:280.

54. Gregory PA, Bert AG, Paterson EL, Barry SC, Tsykin A, Farshid G, Vadas MA, Khew-Goodall Y, Goodall GJ: The miR-200 family and miR-205 regulate epithelial to mesenchymal transition by targeting ZEB1 and SIP1. Nature cell biology 2008, 10(5):593-601.

55. Hurteau GJ, Carlson JA, Spivack SD, Brock GJ: Overexpression of the microRNA hsa-miR-200c leads to reduced expression of transcription factor 8 and increased expression of E-cadherin. Cancer research 2007 67(17):7972-6.

56. Burk U, Schubert J, Wellner U, Schmalhofer O, Vincan E, Spaderna S, Brabletz T: A reciprocal repression between ZEB1 and members of the miR-200 family promotes EMT and invasion in cancer cells. EMBO reports 2008, 9(6):582-9.

57. Peter ME: Let-7 and miR-200 microRNAs: guardians against pluripotency and cancer progression. Cell cycle (Georgetown, Tex.) 2009, 8(6):843-52.

58. Chen L, Li Y, Fu Y, Peng J, Mo M-H, Stamatakos M, Teal CB, Brem RF, Stojadinovic A, Grinkemeyer M, McCaffrey TA, Man Y-g, Fu SW: Role of deregulated microRNAs in breast cancer progression using FFPE tissue. PloS one 2013, 8(1):54213.

59. Luo D, Wilson JM, Harvel N, Liu J, Pei L, Huang S, Hawthorn L, Shi H: A systematic evaluation of miRNA:mRNA interactions involved in the migration and invasion of breast cancer cells. Journal of translational medicine 2013, 11:57

60. Chen Y, Song Y, Wang Z, Yue Z, Xu H, Xing C, Liu Z: Altered expression of MiR-148a and MiR-152 in gastrointestinal cancers and its clinical significance. Journal of gastrointestinal surgery : official journal of the Society for Surgery of the Alimentary Tract 2010, 14(7):1170-9.

61. Zhou X, Zhao F, Wang Z-N, Song Y-X, Chang H, Chiang Y, Xu H-M: Altered expression of miR-152 and miR-148a in ovarian cancer is related to cell proliferation. Oncology reports 2012, 27(2):447-54.

62. Xu Q, Jiang Y, Yin Y, Li Q, He J, Jing Y, Qi Y-T, Xu Q, Li W, Lu B, Peiper SS, Jiang B-H, Liu L-Z: A regulatory circuit of miR-148a/152 and DNMT1 in modulating cell transformation and tumor angiogenesis through IGF-IR and IRS1. Journal of molecular cell biology 2013, 5(1):3-13.

63. Brenner B, Hoshen MB, Purim O, David MB, Ashkenazi K, Marshak G, Kundel Y, Brenner R, Morgenstern S, Halpern M, Rosenfeld N, Chajut A, Niv Y, Kushnir M: MicroRNAs as a potential prognostic factor in gastric cancer. World journal of gastroenterology : WJG 2011, 17(35):3976-85.

64. Pan $X$, Wang $R$, Wang Z-X: The potential role of miR-451 in cancer diagnosis, prognosis, and therapy. Molecular cancer therapeutics 2013, 12(7):1153-62.

doi:10.1186/1471-2105-16-S4-S7

Cite this article as: Fiannaca et al:: Analysis of miRNA expression profiles in breast cancer using biclustering. BMC Bioinformatics 2015 16(Suppl 4): s7. 